\title{
Outcome of Laparoscopic Ovarian Drilling in Patients of Clomiphene Resistant Polycystic Ovarian Syndrome in a Tertiary Care Center
}

\author{
Mandeep Kaur, Gautham Pranesh, Manishi Mittal, Anjali Gahlan, K Deepika, T Shashikala, Kamini A Rao
}

\begin{abstract}
Background: Polycystic ovarian syndrome (PCOS) is the most common endocrine disorder in women and its prevalence is rising. Management of the disease is usually medical and some resistant cases may require surgical treatment in the form of laparoscopic ovarian drilling (LOD). Medical management exposes the patient to increased risk of multiple pregnancy and hyperstimulation. LOD avoids the need of medical therapy or makes the ovaries more responsive to treatment.
\end{abstract}

Aim: The objective of this descriptive study was to study the outcome of LOD in patients of PCOS with clomiphene resistance in the form of clinical pregnancy and live birth rate.

Setting: Tertiary assisted conception center.

Design: Observational study.

Materials and methods: 100 patients of clomiphene resistant PCOS who underwent LOD.

Outcome measure: Primary outcome was clinical pregnancy rate and secondary outcome was ovarian hyperstimulation syndrome (OHSS) rate, multiple pregnancy rate, miscarriage rate, prevalence of hypothyroidism and live birth rate in PCOS patients.

Results: Clinical pregnancy rate- $47.3 \%$, OHSS rate- $2.7 \%$, multiple pregnancy rate-4\%, miscarriage rate-6.7\%, prevalence of hypothyroidism-48\% and live birth rate $-40.5 \%$.

Conclusion: Patients with irregular cycles, high LH/FSH ratio usually have $\mathrm{CC}$ resistance. $\mathrm{PCO}$ patients have high prevalence of hypothyroidism and it should be specifically screened and treated. Low incidence of miscarriage rate, OHSS rate and multiple pregnancy rates is seen after LOD with $47.3 \%$ clinical pregnancy and $40.5 \%$ live birth rates. Patients with high values of $\mathrm{LH} / \mathrm{FSH}$ ratio are the candidates who stay nonpregnant in spite of LOD and this information is very useful in prognosticating the patients.

Keywords: Laparoscopic ovarian drilling, Polycystic ovarian syndrome, Clomiphene, Gonadotropins, Hyperstimulation.

How to cite this article: Kaur M, Pranesh G, Mittal M, Gahlan A, Deepika K, Shashikala T, Rao KA. Outcome of Laparoscopic Ovarian Drilling in Patients of Clomiphene Resistant Polycystic Ovarian Syndrome in a Tertiary Care Center. Int J Infertility Fetal Med 2013;4(2):39-44.

Source of support: Nil

Conflict of interest: None

Date of Received: 06-08-13

Date of Acceptance: 25-08-13

Date of Publication: May 2013

\section{INTRODUCTION}

Polycystic ovarian syndrome (PCOS) is the most common endocrine disturbance to affect women and its prevalence is on an increase. Normal selection of follicle is affected and there are viable oocytes in dysfunctional follicles. In recent times, there is significant change in lifestyle in many parts of the world and people are experiencing abundance of food and lack of physical activity. This could be the reason for the modern epidemic of obesity and hyperinsulinemia which stimulates androgen production causing more early recruitment of follicles but poor cyclic development.

Wedge resection was established as a treatment for anovulatory polycystic ovaries by Stein and Leventhal in $1935^{1}$ but was largely abandoned due to risk of postsurgical adhesions ${ }^{2}$ and with the advent of medicines for ovulation induction. ${ }^{3}$ First choice for ovulation induction is clomiphene citrate and in case of resistance options are gonadotropins or LOD. Clomiphene is successful in $80 \%$ of the cases; the $20 \%$ patients who fail to ovulate are declared clomiphene resistant. ${ }^{4}$

Introduction of LOD (first described by Gjonnaess in $1984)^{5}$ reawakened interest in the surgical management of CC resistant PCOS patients. LOD involves use of cautery or laser vaporization to cause multiple perforations in the ovary. Many studies have claimed an increase in rates of spontaneous ovulation and conception after $\mathrm{LOD}^{6,7}$ along with improved responsiveness to subsequent medical therapy. ${ }^{8}$ LOD can be done on outpatient basis with less trauma and fewer postoperative adhesions. On the other hand, gonadotropins, although effective, expose the patients to risks of multiple pregnancy and hyperstimulation. Also, gonadotropins are expensive and require repeated doses and intensive monitoring.

The objective of this observational study is to review the characteristics of the patients who underwent LOD at our center and to evaluate clinical pregnancy and live birth rates in these patients. Also, its effect on the development of complications like hyperstimulation, miscarriage and multiple pregnancies are taken into account and compared to the data obtained from literature. The characteristics of patients who remained nonpregnant after LOD were analyzed and compared to the pregnant group. We intend 
to assess the effect and safety of the surgery in our group of patients.

\section{MATERIALS AND METHODS}

\section{Study Design and Subject Selection}

The observational study included retrospective data obtained from medical records of patients who underwent LOD at our center between March 2009 and March 2011. As the study was observational, no approval was required from the institutional review board.

\section{Inclusion and Exclusion Criteria}

Criterion for selection is patients with PCOS who were resistant to clomiphene citrate and subsequently underwent LOD. Clomiphene resistance is defined as failure to ovulate in response to even maximum $150 \mathrm{mg}$ of clomiphene. Exclusion criteria were tubal pathology, severe endometriosis and severe male factor. As measurement of androgen levels is not routinely done at our center so it was not a part of workup for PCOS. Only clinical and scan findings were taken into account.

\section{Treatment Protocol}

Laparoscopic ovarian drilling was done using a 'Karl Storz' monopolar drilling needle keeping power at $40 \mathrm{~W}$. Around 4 to 6 punctures were made in each ovary depending on the patient's characteristics. After LOD, as per the protocol of the hospital, patients tried to conceive naturally for 2 months and then were subjected to ovulation induction (OI) with timed intercourse (TI) or intrauterine insemination (IUI) on case to case basis. Patients who failed to conceive by these methods were offered IVF.

\section{Data Collection and Statistical Analysis}

Data for variables, like age, BMI, duration of infertility, body mass index (BMI), LH, FSH, TSH and treatment outcome, were obtained from medical records. Quantitative variables are presented in the form of box and whisker plots. The boxes represent the distribution of the data between the 25th and 75th centiles with the median (line) and mean (diamond) values represented therein. The whiskers represent the minimum and maximum values observed. Qualitative variables are represented as rate percentages. The students t-test was used to evaluate differences between means across groups. A p-value of $<0.05$ was considered as statistically significant. Analysis of data was done on MS Excel 2010.

\section{Outcome Variables}

Clinical pregnancy rate was considered as the primary outcome measure. Clinical pregnancy is defined as ultrasound evidence of fetal heart beat. The rate was expressed as the percentage of patients with clinical pregnancy across the evaluated data set.

Other outcome measures, including live birth rate, miscarriage rate, multiple pregnancy rates and OHSS rate, were calculated in a similar manner.

\section{RESULTS}

We analyzed the baseline characteristics, hormonal profile and outcome of 100 patients who met the inclusion criterion. In total, 35 patients achieved clinical pregnancy, 38 remained nonpregnant and 26 were lost to follow-up (Graph 1). Irregular cycles were seen in $82 \%$ of the patients and $62 \%$ of them had altered LH/FSH ratio ( $\geq 2: 1)$. However, subclinical hypothyroidism was found in $48 \%$ of patients taking a cut off of $2.5 \mathrm{mIU}$ (Tables $1 \mathrm{~A}$ and B).

Out of 74 patients with evaluable data, we observed that three (4.05\%) patients conceived naturally, eight (10.81\%) conceived after OI/TI, 12 (16.21\%) conceived after OI/IUI and seven (9.45\%) had IVF conceptions (Graph 2).

Five (6.75\%) patients had a miscarriage and one (1.35\%) IVF patient had an ectopic pregnancy. None of the patients managed with TI or IUI developed OHSS whereas two (2.7\%) patients in IVF group developed moderate OHSS. None of the patients developed severe or critical OHSS. Twin pregnancies were observed in three (4.05\%) patients in the IVF group (Table 2). Higher order multiple pregnancy was not observed in this study.

After the overall analysis, subgroup analyses of the patients, who got pregnant and who did not, were done and the groups were compared to each other. As can be seen in Table 3, there was no difference between pregnant and nonpregnant patients in their baseline characteristics. There was no statistically significant difference between the TSH values of pregnant and nonpregnant patients $(\mathrm{p}=0.25)$ and no difference in the BMI of the pregnant and nonpregnant patients $(\mathrm{p}=0.39)$.

The basal (day 2-5) FSH and LH levels were not statistically significant in the pregnant and nonpregnant groups with the FSH (p-value - 0.44 ) and LH (p-value — 0.59)

Tables $1 \mathrm{~A}$ and B: Baseline characteristics of patients

\begin{tabular}{lr}
\hline (A) Baseline characteristics & Mean $( \pm S D)$ \\
\hline Age (years) & $27( \pm 3.2)$ \\
Duration of infertility (years) & $4.3( \pm 2.7)$ \\
BMI $\left(\mathrm{kg} / \mathrm{m}^{2}\right)$ & $26.6( \pm 4.2)$ \\
Average LH/FSH ratio & $2.1( \pm 1.1)$ \\
(B) Baseline characteristics & Percentage \\
\hline Irregular cycle & 82 \\
Subclinical hypothyroidism & 48 \\
Altered LH/FSH ratio $(\geq 2: 1)$ & 62 \\
\hline
\end{tabular}


Table 2: Outcome variables studied in the patients

$\begin{array}{lc}\text { Outcome variables } & \text { Percentage } \\ \text { Clinical pregnancy rate } & 47.29 \\ \text { Live birth rate } & 40.54 \\ \text { Miscarriage rate } & 6.75 \\ \text { OHSS rate } & 2.70 \\ \text { Twin pregnancy rate } & 4.05\end{array}$

Table 3: Baseline characteristics of pregnant/nonpregnant patients. Data for variables 'age' and 'duration of infertility' expressed as mean \pm SEM

\begin{tabular}{llr}
\hline Characteristics & Pregnant & Nonpregnant \\
\hline Age (years) & $27.1( \pm 0.46)$ & $26.7( \pm 0.58)$ \\
$\begin{array}{l}\text { Duration of } \\
\text { infertility (years) }\end{array}$ & $3.5( \pm 0.37)$ & $4.48( \pm 0.44)$ \\
$\begin{array}{l}\text { Percentage with } \\
\text { irregular cycles }\end{array}$ & $80.6 \%$ & $78.9 \%$ \\
\hline
\end{tabular}

(Graph 3). On comparison of LH/FSH ratios of pregnant and nonpregnant patients, it was found that the variability of the ratios (p-value-0.17) was higher in nonpregnant patients compared to pregnant patients (Graph 4).

\section{DISCUSSION}

PCOS is a common endocrine disorder with systemic sequelae. It is characterized by oligomenorrhea, signs of androgen excess, obesity and infertility, elevated levels of LH and androgens and not infrequently with insulin résistance. The diagnosis of PCOS requires the presence of at least two of the three criteria, i.e. oligo/anovulation, hyperandrogenism (clinical or biochemical) and ultrasound appearance of polycystic ovaries with exclusion of other etiological factors. ${ }^{9}$ The pathophysiology of PCOS is multifactorial and polygenic.

Surgical treatment of infertility associated with PCOS was first reported by Stein and Leventhal but the procedure

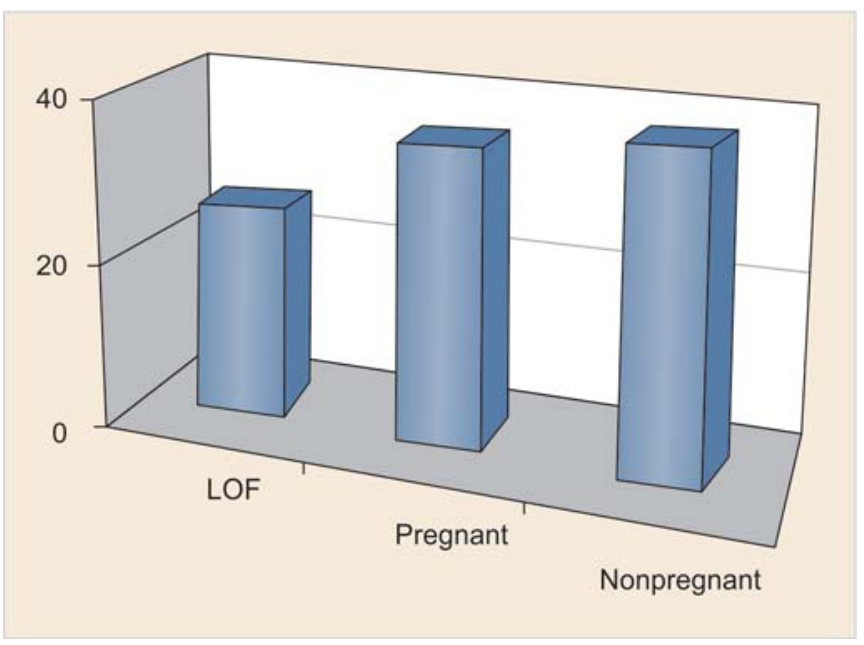

Graph 1: The outcome of patients (LOF-lost to follow-up; Y axis-number of patients) can lead to adhesions and subsequent infertility, so was abandoned. Introduction of LOD reawakened interest in surgical treatment of PCOS, especially in patients

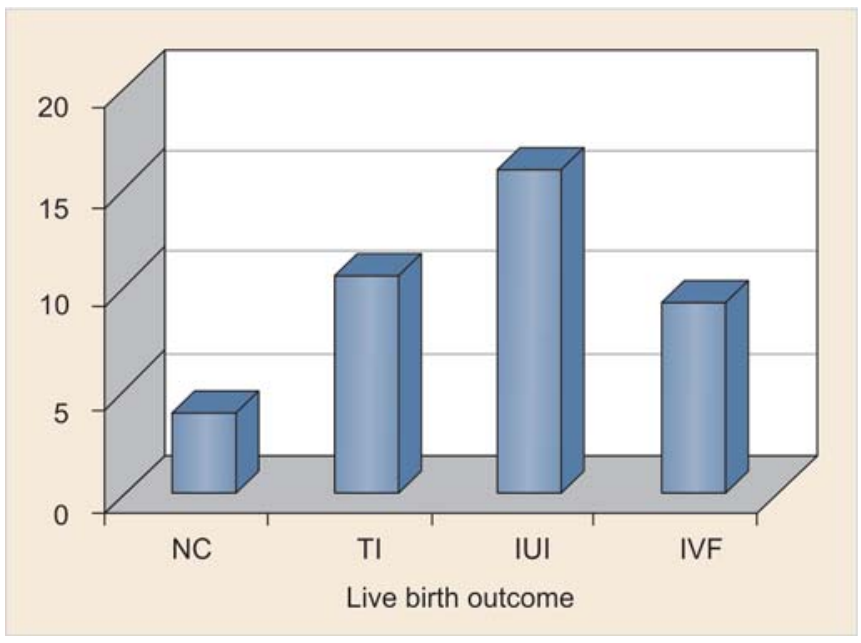

Graph 2: Interventions resulting in pregnancy post LOD. NC indicates natural conception (no intervention); $Y$ axis-percentage

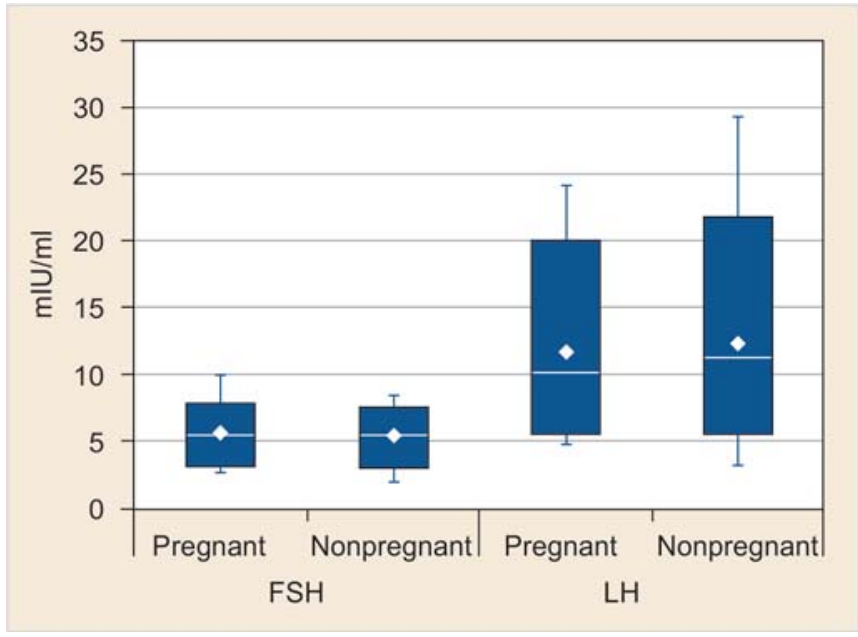

Graph 3: Basal FSH and LH levels of pregnant and nonpregnant patients

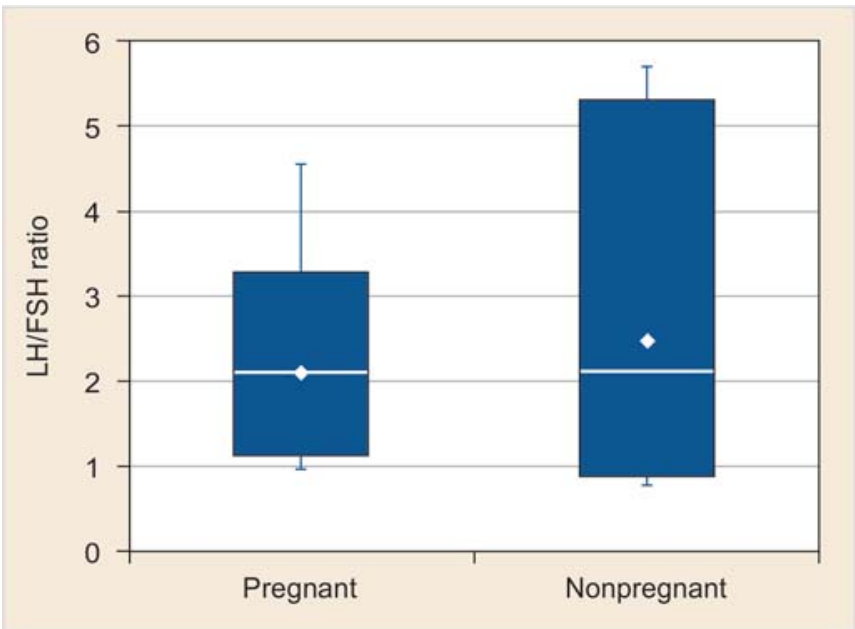

Graph 4: LH/FSH ratios of pregnant and nonpregnant patients 
unresponsive to clomiphene. Other alternatives for clomiphene reistance are gonadotropins, insulin sensitizers, glucocorticoids or aromatase inhibitors. LOD is particularly useful in patients who are not obese and have high LH concentrations. It also benefits patients who live far from the hospital and cannot come for regular follow-up required with gonadotropins.

Ovarian drilling involves the creation of multiple openings in the ovarian capsule using laparotomy, laparoscopy or transvaginal hydrolaparoscopy for surgical access. It can be done with electrosurgery or with laser energy. The ovaries are immobilized, and an insulated electrode is inserted perpendicular to the surface to a depth of 3 to $4 \mathrm{~mm}$.

Menstrual cycle disturbance is a common feature of PCOS. A total of $87 \%$ of women with oligomenorrhea and $26 \%$ of women with amenorrhea presenting in a gynecological clinic have been found to have polycystic ovaries on ultrasound. ${ }^{10}$ In our study, irregular cycles were seen in $82 \%$ of the patients.

Serum LH concentrations are elevated in 40 to $60 \%$ of PCOS patients and elevated concentration is associated with reduced chance of conception and increased risk of miscarriage. ${ }^{11}$ LH levels are higher in lean PCO patients. High LH/FSH ratio is pathognomonic of the disease but is not required to make the diagnosis. It has also been suggested that PCO patients may secrete LH isoforms with high biological activity. It has been observed that patients with high baseline LH levels have better response to LOD.

In our study, $\mathrm{LH} / \mathrm{FSH}$ ratio is altered in $62 \%$ of the patients and average LH/FSH ratio is $2.1( \pm 1.1)$. Average BMI of patients in our study group is $26.6( \pm 4.2)$. Although no statistically significant difference was found in the FSH and LH values of pregnant and nonpregnant patients, an interesting fact pointed out here is that the patients who remained nonpregnant in spite of LOD had high LH/FSH ratios before LOD, pointing toward the high $\mathrm{LH} / \mathrm{FSH}$ ratio as possibly a poor prognostic indicator toward a successful pregnancy after LOD.

The mechanism of LOD is however unclear. Its beneficial effect was proposed to be due to destruction of androgen producing stroma. There is reduction in the intraovarian and circulating levels of androgens and LH. There is also modulating effect on the pituitary ovarian axis. ${ }^{12}$ These endocrine changes occur rapidly and may last for months to years leading to resumption of normal cycles and ovulation. ${ }^{13}$ The additional advantages are that the assessment of tubal patency and diagnosis of other pelvic pathologies can be done simultaneously. Moreover, the sensitivity to ovulation induction increases after the procedure. There is more orderly growth of follicles, reduced chances of cycle cancellation and decreased risk for OHSS. Proper follicular growth occurs in a more favorable environment with low androgen, $\mathrm{AMH}$ and $\mathrm{LH}$ levels and relatively less number of follicles.

As per the Cochrane review in 2012, with 9 RCT and 16 trials, it was found that ovarian drilling is as effective as medical induction of ovulation in terms of clinical pregnancy or live birth, but risk of multiple pregnancy is lower in LOD group. A live birth rate of approximately $37 \%$ is seen in either group with a miscarriage rate of $7 \% .{ }^{14}$ Few studies have obtained clinical pregnancy rates of 43 to $84 \%$. $^{15,16}$ In our study, a clinical pregnancy rate of $47.3 \%$ and live birth rate of $40.3 \%$ were obtained.

Multiple pregnancy rate of $4 \%$ (3 patients) was seen in our study. No patients in the IUI or TI groups developed multiple pregnancies. Only three patients in the IVF group developed twin conception. Number of embryos transferred could be a confounding variable. As mentioned in the Cochrane database review, the average range of multiple pregnancies in the patients after LOD is 0 to $10 \%$ but is significantly lower than the gonadotropins. ${ }^{14}$

PCOS patients are prone to development of OHSS and high levels of VEGF and IGF-1 have been implicated in its causes. LOD decreases the risk of development of OHSS after the procedure due to fall in levels of VEGF. In our study, none of the patients developed severe or critical OHSS. Only two patients in the IVF group developed moderate OHSS (2.7\%).

It has been observed that PCOS patients have higher miscarriage rates as compared to the general population. It was also postulated that LH is the culprit. Hyperinsulinemia and obesity are also known to be contributory in the increased miscarriage rates in PCOS patients. ${ }^{11}$ LOD causes reduction in LH levels and reduces the risk of miscarriage. In a study, miscarriage rate of $14 \%$ has been reported after LOD as compared to 30 to $40 \%$ expected rate in PCOS patients. ${ }^{17} \mathrm{~A}$ miscarriage rate of $6.7 \%$ was seen in our study.

However, there are concerns with the procedure like the risks involved with surgery, the development of adhesions and the risk of ovarian failure following LOD. No immediate complications of surgery or anesthesia were seen in any of our patients pointing toward the safety of the procedure. As far as the cost is concerned, LOD adds 20 minutes to the routine diagnostic laparoscopy adding to the cost, but, if the indirect cost of multiple pregnancy and cycle cancellations is considered, it seems to be reasonably cost effective.

The likelihood of injury to the ovary is very low with LOD, but the hilar region should be avoided and ovary should be raised before application of energy and saline 
wash should be done after the procedure to lower the temperature, decreasing risk of injury. Greater the amount of damage to the surface of the ovaries, greater is the risk of adhesion formation. This led to development of a strategy of applying four diathermy points to each ovary for 4 seconds each and using power of $40 \mathrm{~W} .^{18}$

As second look laparoscopy and adhesiolysis is of no use in improving fertility, it was not done in our patients. However, the adhesion formation after LOD is usually mild and is less frequent compared to conventional wedge resection.

In our study, the prevalence of subclinical hypothyroidism is seen to be $48 \%$ using a cut off of $2.5 \mathrm{mIU} / \mathrm{ml}$. So with such high prevalence, it is very important to screen patients of PCOS for thyroid function tests and treat them accordingly. Thyroid pathologies are observed in half of the patients with PCOS. Measurement of thyroid hormones should be a part of investigation in PCOS patients, but serum thyroid autoantibodies and presence of thyroid nodules should also be searched in these patients. ${ }^{19}$ Hypothyroidism is invariably followed by a lowering of sex hormone binding globulin and an increment in the free testosterone level and may have implications in the development of PCOS in the hypothyroid state. ${ }^{20}$

It has also been reported that the incidence of gestational diabetes mellitus decreases in pregnancies that occur after $\mathrm{LOD}^{21}$ and an incidence of $8 \%$ has been reported compared to expected rate of $20 \%$ in untreated PCOS patients. A study has also mentioned that incidence of gestational diabetes does not improve in spite of any treatment. ${ }^{22}$ However, this has not been studied in our patients and has scope for future research.

\section{CONCLUSION}

Ovulation induction in PCOS patients is the solution for infertility after all other causes are ruled out. Clomiphene citrate is the drug of choice but $20 \%$ of patients have clomiphene resistance. Adjuvant drugs like metformin, aromatase inhibitors, myo-inositol and steroids may be tried before resorting to LOD. In case of persistent resistance, two options are available-gonadotropins or LOD. Case selection is very important to decide mode of treatment. Clinical pregnancy and live birth rates are reasonable and along with that there is reduction in miscarriage, OHSS and multiple pregnancy rates with LOD. It also has the advantage of being a day care procedure with minimal complications. Tubal patency and anatomy of pelvic organs can be seen simultaneously. However, it has to be performed by a trained surgeon with proper case selection to avoid any complications.

Patients with irregular cycles and high LH/FSH ratio usually have CC resistance. PCOS patients have high prevalence of subclinical hypothyroidism and it should be specifically screened and treated. Low miscarriage, OHSS and multiple pregnancy rates are seen after LOD with reasonably good clinical pregnancy and live birth rates. High values of LH/FSH ratio point toward lesser chances of pregnancy in spite of LOD and this information may be useful in prognosticating patients. However, more studies are required before establishing this as a definite prognostic factor. A cut off can be established with higher powered studies that help to decide the treatment plan in PCOS.

\section{REFERENCES}

1. Stein IF, Cohen MR. Surgical treatment of bilateral polycystic ovaries. Am J Obst and Gynae 1939;38:465-473.

2. Adashi N, Rock JA, Guzick D, Wentz AC, Jones GS, Jones HW Jr. Fertility following bilateral ovarian wedge resection: critical analysis of 100 consecutive cases of PCOS. Fertility and Sterility 1981;36:30-35.

3. Franks S, Adams J, Mason H, Polson D. Ovulation disorders in women with polycystic ovarian syndrome. Clinical Obst and Gyne 1985;12:605-633.

4. Imani B, Eijkemans MJC, teVelde ET, Habbema JDF, Fauser BCJM. Predictors of patients remaining anovulatory during clomiphene induction of ovulation in normo-gonadotropic oligoamenorrhic infertility. J Clinic Endocrin and Metabol 1998; 83(7):2361-2365.

5. Gjonnaess. Polycystic ovarian syndrome treated by ovarian electrocautery through the laparoscope. Fertility and Sterility 1984;49:956-960.

6. Greenbalt E, Casper RF. Endocrine changes after laparoscopic ovarian cautery in polycystic ovarian syndrome. Am J Obste and Gynae 1987;156:279-285.

7. Kovacs G, Buckler H, Bangah M, Outch K, Burger H, Healy D. Treatment of anovulation due to polycystic ovarian syndrome by laparoscopic ovarian electro-cautery. British J Obste and Gynae 1991;98:30-35.

8. Farhi J, Soule S, Jacobs HS. Effect of laproscopic ovarian electrocautery on ovarian response and outcome of treatment with gonadotropins in clomiphene resistant patients with polycystic ovaries. Fertility and Sterility 1995;64:930-933.

9. The Rotterdam ESHRE/ASRM-sponsered PCOS consensus workshop group. Revised 2003 consensus on diagnostic criterion and long term health risks related to PCOS. Hum Reprod 2004; 19:41-47.

10. Adams J, Polson DW, Franks S. Prevalence of polycystic ovaries in women with anovulation and idiopathic hirsutism. Br Med J 1986:293:355-359.

11. Homburg R. Pregnancy complications in PCOS. Best Pract Res Clin Endocrinol Metab 2006 Jun;20(2):281-292.

12. Rossmanith WG, Keckstein J, Spatzier Klauritzen C. The impact of ovarian laser surgery on the gonadotropin secretion in women with polycystic ovarian disease. Clin Endocrinol (oxf) 1991;34: 223-230.

13. Amer SA, Gopalan V, Li TC, Ledger WL, Cookie ID. Longterm follow-up of polycystic ovary disease after laparoscopic ovarian drilling: clinical outcome. Hum Reprod 2002;17: 2035-2042. 
14. Farquhar C, Brown J, Marjoribanks J. Laparoscopic drilling by diathermy or laser for ovulation induction in anovulatory polycystic ovary syndrome. Cochrane Database Syst Rev 2012 Jun 13;6:CD001122.

15. Campo S, Felli A, Lamanna MA, Barini A, Gracea N. Endocrine changes and clinical outcome after laparoscopic ovarian resection in women with polycystic ovaries. Hum Reprod 1993;8:359-363.

16. Gjonnaess H. Late endocrine effects of ovarian electrocautery in women with polycystic ovary syndrome. Fertil Steril 1998;69: 697-701.

17. Armar NA, Lanchelin GCL. Laparoscopic ovarian diathermy: an effective treatment for anti-estrogen resistant anovulatory infertility in women with polycystic ovaries. Br J Obset Gynaecol 1993;100:161-164.

18. Armar NA, McGarrigle HHG, Honour JW, Holownia P, et al. Laparoscopic ovarian diathermy in the management of anovulatory infertility in women of polycystic ovaries: endocrine changes and clinical outcome. Fertil Steril 1990;53:45-49.

19. Ozdemir D, Cuhaci N, Balkan F, Usluogullari A, Ersoy R, Cakir B. Prevalence of thyroid pathologies in patients with polycystic ovary syndrome. Endocrine Abstracts 2011;26:92.

20. Ghosh S, Kabir SN, Pakrashi A, Chatterjee S, Chakravarty B. Subclinical hypothyroidism: a determinant of polycystic ovary syndrome. Hormone Research in Paediatrics 1993;39:1-2.

21. Gjonnaess H. The course and outcome of pregnancy and of pregnancy after ovarian electrocautery in women with polycystivc ovarian syndrome: the influence of body-weight. Br J Obstet Gynaecol 1989;96:714-719.

22. Ott J, Kurz C, Nouri K, Wirth S, Vytiska-Binstorfer E, Huber JC, Mayerhofer K. Pregnancy outcome in women with polycystic ovary syndrome comparing the effects of laparoscopic ovarian drilling and clomiphene citrate stimulation in women pre-treated with metformin: a retrospective study. Reprod Biol Endocrinol 2010 May 13;8:45.

\section{ABOUT THE AUTHORS}

\section{Mandeep Kaur (Corresponding Author)}

Fellow, Department of Reproductive Medicine, Bangalore Assisted Conception Centre, Bengaluru, Karnataka, India, Phone: 08884838538 e-mail: docmandeep80@gmail.com

\section{Gautham Pranesh}

Consultant, Department of Reproductive Medicine, Bangalore Assisted Conception Centre, Bengaluru, Karnataka, India

\section{Manishi Mittal}

Fellow, Department of Reproductive Medicine, Bangalore Assisted Conception Centre, Bengaluru, Karnataka, India

\section{Anjali Gahlan}

Fellow, Department of Reproductive Medicine, Bangalore Assisted Conception Centre, Bengaluru, Karnataka, India

\section{K Deepika}

Consultant, Department of Reproductive Medicine, Bangalore Assisted Conception Centre, Bengaluru, Karnataka, India

\section{T Shashikala}

Consultant, Department of Reproductive Medicine, Bangalore Assisted Conception Centre, Bengaluru, Karnataka, India

\section{Kamini A Rao}

Medical Director, Department of Reproductive Medicine, Bangalore Assisted Conception Centre, Bengaluru, Karnataka, India 